\title{
Errata: Correlating the amount of urea, creatinine, and glucose in urine from patients with diabetes mellitus and hypertension with the risk of developing renal lesions by means of Raman spectroscopy and principal component analysis
}

Jeyse Aliana Martins Bispo

Elzo Everton de Sousa Vieira

Landulfo Silveira, Jr.

Adriana Barrinha Fernandes 


\title{
Errata: Correlating the amount of urea, creatinine, and glucose in urine from patients with diabetes mellitus and hypertension with the risk of developing renal lesions by means of Raman spectroscopy and principal component analysis
}

\author{
Jeyse Aliana Martins Bispo, ${ }^{a, b}$ Elzo Everton de Sousa Vieira, ${ }^{a, b}$ Landulfo Silveira Jr., and \\ Adriana Barrinha Fernandes ${ }^{a}$ \\ aUniversidade Camilo Castelo Branco-UNICASTELO, Biomedical Engineering Institute, Parque Tecnológico de São José dos Campos, \\ Estr. Dr. Altino Bondesan, 500, São José dos Campos, São Paulo 12247-015, Brazil \\ ${ }^{6}$ Faculdades Integradas do Tapajós-FIT, Rua Rosa Vermelha, 335, Aeroporto Velho, Santarém, Pará 68010-200, Brazil
}

[DOI: 10.1117/1.JBO.21.5.059801]

This article [J. Biomed. Opt. 18(8), 087004 (2013)] was originally published online on 8 August 2013. Numerous textual details were changed on p. 6, and the last eight paragraphs of Sec. 4 are reprinted here. The article was corrected online on 26 April 2016.

According to Basto and Kirsztajn, ${ }^{10}$ optimal management of chronic kidney disease (CKD) is based on three pillars: (1) early diagnosis of disease, (2) immediate referral for nephrological treatment, and (3) implementation of measures to preserve renal function. The early diagnosis of disease is frequently difficult because of the absence of symptoms in patients in the early stages of CKD, thus requiring that clinicians may suspect all patients, especially for those with risk factors for CKD.

Functional change in the GFR is an important characteristic in the diagnosis and classification of CKD. ${ }^{10}$ Even with reduced number of nephrons, the stable or near-normal GFR can be explained by the occurrence of increased filtration pressure or glomerular hypertrophy. This is sometimes observed in early diabetic nephropathy, where the GFR is increased up to $40 \%$ above the normal value. ${ }^{28,29}$

There is no evidence that the GFR is lessened by any kind of strict glycemic control. ${ }^{6,7,9}$ On the other hand, HT has been found to be a major factor in the prediction of progression of diabetic nephropathy along with microalbminuria and hyperglycemia. ${ }^{30}$

The best way to evaluate the GFR is by determining the clearance of exogenous substances such as inulin, 125I-iothalamate, ethylenediaminetetraacetic acid, technetium-labeled diethylene triamine pentaacetic acid, or iohexol. As these compounds are excreted from the body via glomerular filtration, and they do not undergo further secretion and/or reabsorption when passing through the renal tubules, they are considered an ideal filtration marker. ${ }^{31}$ Since these exogenous substances need to be infused, the evaluation of these clearances is challenging, requiring a long time for the procedure, and has been restricted to specific pathological conditions or research, where more simple clearance techniques do not provide sufficient information to guide the medical decision. ${ }^{10}$

In clinical practice, the GFR is assessed by measuring specific endogenous biomarkers such as urea and creatinine. Urea is not completely reliable since its levels are susceptible to changes

(C) 2016 Society of Photo-Optical Instrumentation Engineers (SPIE) due to other conditions not related to GFR. For instance, a high protein diet, intense gastrointestinal hemorrhage, tissue breakdown, and therapy with corticosterols can lead to an increase in plasma urea, whereas a low protein diet and liver disease can lead to its reduction. Also, since $40 \%$ to $50 \%$ of filtered urea may be reabsorbed by the tubules, this proportion is reduced in advanced renal failure. ${ }^{32,33}$

The most used method for obtaining information on GFR is the creatinine clearance in 24-h urine, in which the urinary creatinine excretion is divided by the serum creatinine concentration. The creatinine clearance does not present all the criteria for an ideal marker of GFR since creatinine is excreted via glomerular filtration and also via secretion in the proximal tubule. ${ }^{32-34}$ The major problem with creatinine clearance is the requirement for urine collection over $24 \mathrm{~h}$, where the collections are often inaccurate, particularly in some clinical situations (elderly patients, cognitive impairment). At present, determination of GFR by creatinine clearance is recommended even in extremes of age and body size, obesity, severe malnutrition, vegetarian diet, disease of skeletal muscle, paraplegia or quadriplegia, acute renal failure, and adjustment of dosage of potentially nephrotoxic drugs. ${ }^{32,33}$

The course of CKD is often asymptomatic until the disease reaches its advanced stages, usually diagnosed when the patient seeks medical attention after presenting one or more disease complications and/or other comorbidities such as DM and HT. It remains unclear which patients with CKD will progress to end-stage renal disease and which ones are at greater risk of needing renal dialysis. However, it is important that interventions would be implemented earlier for stabilizing the progression of renal disease and preventing the occurrence of end-stage renal disease. Furthermore, it is important to emphasize that successful treatment of the underlying disease, such as DM and HT, is also needed for preventing the end-stage renal disease. ${ }^{10}$

Early diagnosis and immediate nephrology referral are key steps in management because enable predialysis education, allow implementation of preventive measures that delay or even halt progression of CKD to end-stage renal disease, as well as decrease initial morbidity and mortality. ${ }^{10}$ Thus, Raman spectroscopy is a rapid and reliable method that can be useful in future diagnosis of complications from DM and HT by evaluating the amount of urea, creatinine, and glucose in single urine samples and using them to prevent complications. 